\title{
A Novel Grape Downy Mildew Resistance Locus from Vitis rupestris
}

\author{
Gaurab Bhattarai, ${ }^{1}$ Anne Fennell, ${ }^{2}$ Jason P. Londo, ${ }^{3}$ Courtney Coleman, ${ }^{1}$ \\ and Laszlo G. Kovacs ${ }^{1 *}$
}

\begin{abstract}
The viticulture industry needs advanced grape cultivars with genes that enhance disease resistance and environmental stress tolerance to meet the challenges of a changing climate. To discover beneficial allelic variants of grape genes, we established an $\mathrm{F}_{1}$ mapping population from a cross between two North American grapevines, Vitis rupestris Scheele and Vitis riparia Michx. We generated genotyping-by-sequencing (GBS) markers and constructed parental linkage maps consisting of 1177 and 1115 GBS markers, respectively (LOD threshold $\geq 14$ ), which were validated by mapping the sex-determining locus to chromosome 2. Taking advantage of loci heterozygous in both parents, we also constructed an integrated map containing 2583 markers. We mapped a major quantitative trait locus (QTL) for downy mildew (Plasmopara viticola) resistance to chromosome 10 of $V$. rupestris using both greenhouseand in vitro-generated leaf resistance data. This QTL explains $66.5 \%$ of the phenotypic variance under greenhouse conditions, and its 2-LOD confidence interval corresponds to region 2,470,297 to 3,024,940 bp on chromosome 10 in the Vitis vinifera L. PN40024 reference genome sequence (assembly 12X.v2). We provide PN40024-projected positions of the GBS markers, which can be used as anchors to develop additional markers for the introgression of this $V$. rupestris haplotype into cultivated grape varieties.
\end{abstract}

Key words: disease resistance, linkage map, Plasmopara viticola, QTL, Vitis riparia, Vitis rupestris

Grape (Vitis vinifera L.) cultivation relies heavily on the recurrent application of fungicides, a disease-control method that is both costly and potentially harmful to the environment and human health. Cultivation of disease-resistant grape varieties carrying genes that enhance defense against pathogens is an approach that helps reduce the amount of fungicides applied in viticulture. Traditionally, the source of such defense-related genes has been the North American wild relatives of $V$. vinifera, as they coevolved with now-pandemic pathogens and acquired allelic diversity that strengthens resistance against fun-

\footnotetext{
${ }^{1}$ Department of Biology, Missouri State University, Springfield, MO 65897; ${ }^{2}$ Department of Agronomy, Horticulture \& Plant Science, South Dakota State University, Brookings, SD 57007; and ${ }^{3}$ Grape Genetics Research Unit, USDAAgriculture Research Service, Geneva, NY 14456.

*Corresponding author (Laszlokovacs@missouristate.edu; tel: 417-429-3862) Acknowledgments: The authors thank John Heywood, Zoë Migicovsky, Avinash Karn, Allison Miller, Laura Klein, Daniel Wilkinson, Sonu Koirala BK, and Mani Awale for their help with various aspects of this work. This work was supported by funds from the National Science Foundation Plant Genome Research Program (Award No. 1546869), the USDA-NIFA Specialty Crop Research Initiative (Award No. 2011-51181-30635), the South Dakota Agricultural Experiment Station, Hatch Project No. SD00H633-18, and the Missouri State University Graduate College.

Supplemental data is freely available with the online version of this article at www.ajevonline.org.

Manuscript submitted May 2020, revised Aug 2020, accepted Aug 2020

This is an open access article distributed under the CC BY license (https:// creativecommons.org/licenses/by/4.0/).

By downloading and/or receiving this article, you agree to the Disclaimer of Warranties and Liability. The full statement of the Disclaimers is available at http://www.ajevonline.org/content/proprietary-rights-notice-ajev-online. If you do not agree to the Disclaimers, do not download and/or accept this article. doi: 10.5344/ajev.2020.20030
}

gal and oomycete diseases (Alleweldt and Possingham 1988). Grapevine breeding has a long history of introgressing genetic information from wild grapevines (Vitis species) into $V$. vinifera. The first interspecific grapevine crosses were made in the United States during the early and mid-19th century, followed by a surge of breeding activity in Europe in the wake of the phylloxera epidemic in the late 19th and early 20th centuries (Reisch et al. 2012). This pioneering work focused on developing disease-resistant, fruit-producing varieties through crosses between $V$. vinifera and North American grape species and on breeding phylloxera- and lime-tolerant rootstocks through interspecific crosses among various American grapevines. These efforts met with great success, to the extent that hybrids from the late 19th century are still popular fruit-producing varieties in the eastern and midwestern US. Several of the interspecific American hybrids are among the most widely used rootstocks worldwide today (Di Gaspero et al. 2012, Migicovsky et al. 2016, Riaz et al. 2019).

While introgression of disease resistance traits continued through the 20th century, exploration of wild grape relatives for new resistance sources has lagged. Most breeding work during the past century focused on germplasm that was introduced to Europe from North America during the 1800s. This resulted in a narrow genetic base for both fruit-producing (Di Gaspero et al. 2012) and rootstock (Riaz et al. 2019) hybrids. The high degree of polymorphism reported in North American wild grapevines (Liang et al. 2019) suggests that the genetic diversity of this germplasm is vastly more extensive than what is represented in hybrid cultivars today. Recent examples in which a broader exploration of this germplasm led to the discovery and deployment of valuable haplotypes include the introgression of Pierce's disease resistance from 
Vitis arizonica (Riaz et al. 2009) and powdery and downy mildew resistance from Muscadinia rotundifolia (Feechan et al. 2013, Agurto et al. 2017).

In this paper, we describe linkage map construction and quantitative trait locus (QTL) analysis in an $\mathrm{F}_{1}$ family from a cross between Vitis rupestris and Vitis riparia, two species that vary in habitat and in adaptation to different environmental conditions. The seed parent used in this cross is $V$. rupestris B38, which was collected by Herbert C. Barrett in Texas in 1951 and donated to the National Germplasm Repository in Geneva, NY (PI588160) by Bruce Reisch in 1985. The pollen parent is $V$. riparia HP-1, which was collected by Neils E. Hansen in Bismarck, ND and donated to the National Germplasm Repository in Geneva, NY (PI588271) by Ronald Peterson in 1987. V. rupestris forms shrubs that sprawl along the surface of nutrient-poor sand or gravel bars in intermittent streams or stony outcroppings and grows in small populations within a limited geographic area. $V$. riparia, a sister taxon of $V$. rupestris, forms high-climbing lianas in moist, but well-drained, alluvial soils along rivers and thrives in large populations across great expanses of the continent. As these evolutionarily closely-related grapevine species (Klein et al. 2018) have adapted to contrasting environmental conditions in their native habitats, their alleles will likely influence horticultural traits in different ways. The parent plants were selected because they have large differences in fall photoperiod response, which is likely tied to cold tolerance, and because we wanted to pseudo-replicate the specific cross that produced the commercial rootstock 3309C. We expected to find in the $\mathrm{F}_{1}$ hybrid progeny of this cross abundant potential in segregation for other important viticultural traits, such as branching, angle of growth, leaf shape, root growth, periderm formation, and disease resistance. We hypothesized, therefore, that their $\mathrm{F}_{1}$ hybrid progeny would allow mapping of economically relevant genomic loci.

\section{Materials and Methods}

Mapping population. An $\mathrm{F}_{1}$ mapping population was developed in 2014 by crossing $V$. rupestris accession PI588160 (female parent) with $V$. riparia accession PI588271 (male parent) (Germplasm Resources Information Network 2019). Crosses were made in the field by manually removing floral caps on the $V$. rupestris parent and applying dried collected pollen from the $V$. riparia parent. Inflorescences were covered with paper bags for three weeks to prevent unintended pollination. Seeds were collected from berries (fully colored and soft), vernalized four weeks at $4^{\circ} \mathrm{C}$, and germinated under greenhouse conditions in a 1:1 mix of PRO-MIX and perlite. In spring 2015, seedlings were planted in the vineyard nursery and grown without irrigation or fertilizer, using pest management treatments as needed for powdery mildew, downy mildew (DM), black rot, phomopsis, and anthracnose. In 2018, the surviving seedlings $(\mathrm{n}=257)$ were transplanted into a permanent research vineyard at the USDA clonal germplasm repository in Geneva, NY (42.89'N; 77.00'W). An additional 100 seedlings were germinated and maintained as potted plants at South Dakota State University as part of a pheno- typing project. The combined 357 vines were genotyped for the development of the genetic maps.

Genotyping. A young leaf was collected from each vine into a well of a 96-well plate, frozen promptly, and stored in a $-80^{\circ} \mathrm{C}$ freezer until processing. Two grinder beads were placed into each tube and leaf tissue was ground using a Geno/Grinder 2000 (OPS Diagnostics LLC). Genomic DNA was extracted from each $\mathrm{F}_{1}$ progeny plant and parents with DNeasy 96-well DNA extraction kits (Qiagen). Genotypingby-sequencing (GBS) was performed following a previously described protocol design (Elshire et al. 2011) with modification (Hyma et al. 2015). Barcoded adapters were ligated for each individual sample and single-end sequencing of $100 \mathrm{bp}$ was performed using HiSeq 2000 (Illumina Inc.) at the Institute of Biotechnology, Genomics Facility at Cornell University in Ithaca, NY. Illumina reads were submitted to the NCBI BioSample Database (SAMN13512746 through SAMN13513110). The raw reads were demultiplexed, parsed, and trimmed for quality. Processed reads were aligned using BWA version 0.6.2-r126 (Li and Durbin 2009) against the 12X.v2 V. vinifera PN40024 reference genome sequence (RefSeq) (Jaillon et al. 2007, Canaguier et al. 2017). SNP (single nucleotide polymorphism) genotypes were called using TASSEL-GBS pipeline version 3.0.139 (Glaubitz et al. 2014).

Marker generation and map construction. GBS reads that aligned to the $V$. vinifera RefSeq were screened for SNPs. The identified SNPs were filtered using VCFtools v0.1.13 (Danecek et al. 2011) to retain only biallelic SNPs at a sequencing depth of $\geq 6$. Further, only SNPs with missing genotypes of $\leq 20 \%$ and with minor allele frequency of $\geq 0.2$ were retained. The resulting SNP data in the VCF file were then converted to JOINMAP 5.0 (Van Ooijen 2006) format using NGSEP (Duitama et al. 2014). $F_{1}$ genotypes with more than $10 \%$ missing SNP markers were discarded and a goodness-of-fit $\left(\chi^{2}\right)$ test was performed to filter out test-cross and intercross markers deviating from the 1:1 and 1:2:1 segregation ratio in the progeny, respectively. Because segregation distortion is a natural phenomenon in outcrossing species such as grape, markers showing a moderate degree of segregation distortion were retained for the map construction and only significantly distorted markers $(p<0.0005)$ were discarded. Identical markers were identified and removed from the analysis. Maternal and paternal population nodes were created in JOINMAP 5.0 with marker types " $11 \times 1 \mathrm{~m}$ " and " $\mathrm{nn} \times \mathrm{np}$ ", respectively, and parental maps were constructed following the two-way pseudo-test cross approach (Grattapaglia and Sederoff 1994). Only second-round maps were accepted for each parent, using the default jump threshold of five to maximize the number of markers included in the maps, while limiting inclusion of markers with weak linkage. Markers of the "hk $\times \mathrm{hk}$ " type were then used to integrate parental linkage maps into a consensus map. Each linkage group was constructed with a threshold logarithm of odds (LOD) value of 14 , maximum recombination frequency of 0.4 , and jump threshold of 5. Marker order was determined with a regression mapping algorithm and genetic distances were expressed in Kosambi map units with parameters at default settings. Linkage maps 
were visualized using the software LinkageMap View (Ouellette et al. 2018).

Phenotyping the $\mathrm{F}_{1}$ progeny for $\mathrm{DM}$ resistance. In the greenhouse, phenotyping was carried out by quantifying DM resistance on naturally infected leaves of two replicate plants for each of $136 \mathrm{~F}_{1}$ genotypes five days after symptoms first appeared. Disease developed on naturally infected plants in the greenhouse, was monitored during development, then evaluated at a single time point when shoots were at the eight- to 10-node stage. Scoring was performed using a disease resistance scale of 1 to 10 , where 1 represented the greatest susceptibility $(100 \%$ of leaves had $>50 \%$ of the leaf area on the abaxial side covered with sporangiophores) and 10 the greatest resistance (all leaves had minimal or no sporangial growth). All leaves on a shoot were used to provide the score (coverage over the entire shoot). Plants were then stripped and pruned to two buds and sprayed with Dithane.

An in vitro disease assay was performed to determine if the symptoms seen in the greenhouse were reproducible under more tightly controlled conditions. The 86 individuals phenotyped in vitro were part of the same $F_{1}$ population as those phenotyped following natural infection, but only 20 individuals were shared between the two cohorts. Healthy leaves from the third and fourth nodes from the apical meristem were surface-sterilized in $1 \% \mathrm{NaOCl}$ solution for $2 \mathrm{~min}$ and then rinsed four times in sterile deionized water $\left(\mathrm{dH}_{2} \mathrm{O}\right)$ for $5 \mathrm{~min}$ per rinse. Four circular leaf disks, $2 \mathrm{~cm}$ in diameter, were excised from each leaf and placed abaxial-side up on $0.8 \%$ water-agar plates in petri dishes. DM was collected from infected leaves in the greenhouse and propagated on susceptible leaves to amplify the inoculum. A sporangial suspension of Plasmopara viticola was prepared by suspending sporangia in $\mathrm{dH}_{2} \mathrm{O}$ at a density of 70,000 sporangia/mL, which was then sprayed over the leaf disks uniformly. Inoculated leaf disks were incubated overnight in darkness under axenic conditions and transferred to a growth chamber set at $21^{\circ} \mathrm{C}$ with a 5 -hr/19-hr dark/light diurnal cycle. The leaf disks were scored visually for disease resistance seven days after inoculation. Leaf surface area coverage was estimated using OIV standard disease resistance chart 452 (International Organization of Vine and Wine 2009), which uses a scale of 1 to 9, where 1 and 9 represent the greatest susceptibility and greatest resistance, respectively (Supplemental Figure 1). Each leaf disk was evaluated by two observers independently and their scores were averaged. Though the scales of the greenhouse and in vitro phenotyping had slightly different grading, they both had the same direction.

Characterization of the DM strain. To characterize the $P$. viticola strain responsible for this infection (named MO-1), the genomic DNA of the pathogen was extracted by boiling sporangiophores in the presence of 5\% Chelex, and a 235 bp-long internal transcribed spacer-1 (ITS-1) sequence of the 5.8 S ribosomal RNA gene was PCR-amplified using a specific ITS-1 primer pair (Rouxel et al. 2014). The PCR product was then sequenced and aligned to the corresponding ITS nucleotide sequence of other $P$. viticola cryptic species. To assess the virulence of MO-1 on different grapevine species, the in vitro disease assay was performed using leaf disks of three different grapevines, $V$. riparia Gloire de Montepellier, M. rotundifolia Thomas, and $V$. vinifera F2-35, plus the parents of the $F_{1}$ population. Leaf surface area coverage was estimated using the 1-to-9 scale of the OIV standard disease resistance chart 452 (International Organization of Vine and Wine 2009).

QTL analysis. QTL analysis was performed in MapQTL 6.0 (Van Ooijen 2009) using the integrated map. The interval mapping method was applied to detect significant associations between phenotypic traits and markers using a regression approach. Genome-wide LOD thresholds $(p<0.05)$ were determined for each phenotype by performing 1000 permutations. The genetic regions for significant LOD peaks were identified with corresponding 2-LOD intervals, the predicted gene content in this region was identified using the most recent annotation of the RefSeq (Grimplet et al. 2012, Canaguier et al. 2017), and the percentage of phenotypic variance explained by each QTL was calculated. QTL graphs were generated in MapChart version 2.32 (Voorrips 2002).

\section{Results}

Linkage map construction. The removal of $F_{1}$ individuals with $>10 \%$ missing data reduced the number of individuals in the mapping population to 294 . Filtering 348,888 SNPs across this population for various quality parameters yielded 11,063 SNPs. Of the SNPs that satisfied the filtering criteria, 3436 were discarded because both parents were homozygous for these sites. An additional 1276 sites with unexpected genotypes were excluded from downstream analysis. First, "1l $\times$ $1 \mathrm{~m}$ "- and " $n n \times$ np"-type SNP markers were used to construct parental maps. Population nodes were created in JOINMAP 5.0 for each parent separately. An additional 331 and 360 markers were removed from the maternal and paternal nodes, respectively, because their segregation was distorted from the expected 1:1 ratio as determined by $\chi^{2}$ test $(p<0.0005)$. Upon the removal of identical markers from each parental node, 1462 female parent- and 1351 male parent-informative markers following " $11 \times 1 \mathrm{~m}$ " and " $\mathrm{nn} \times \mathrm{np}$ " segregation types were used for linkage map construction. For the female and male parents, 1177 and 1115 significant markers (LOD threshold $\geq 14$ ) were grouped into 19 different linkage groups covering $1401.3 \mathrm{cM}$ and $1657.4 \mathrm{cM}$ of genetic distance (Table 1), respectively. Linkage groups were numbered according to the assignment of $V$. vinifera RefSeq chromosome map-anchored SNP markers. For $V$. rupestris, the number of SNP markers on each linkage group varied, from a maximum of 114 on LG14 to a minimum of 31 on LG6. The longest and shortest linkage groups for $V$. rupestris were LG18 $(108.7 \mathrm{cM})$ and LG6 (58.6 cM), respectively. In $V$. riparia, LG7 and LG10 had the most (92) and fewest (33) SNP markers, respectively, and LG18 $(125.1 \mathrm{cM})$ and LG9 $(63 \mathrm{cM})$ were the longest and shortest linkage groups, respectively. Although the female map contains more markers than the male map, it spans a shorter genetic length. Furthermore, 291 "hk $\times$ hk"- type markers were combined with the male and female maps to construct an integrated map. The integrated linkage map consists of 2583 
markers distributed on 19 linkage groups and spans a genetic distance of $1634.1 \mathrm{cM}$, with an average marker interval of $0.63 \mathrm{cM}$ (Supplemental Figure 2). Synteny between marker genetic positions on the linkage maps and their corresponding physical coordinates in the RefSeq are shown (Supplemental Figure 3). The detailed genotype information for each marker across $294 \mathrm{~F}_{1}$ progeny were compiled for the $V$. rupestris, $V$. riparia, and integrated maps (Supplemental Tables 1, 2, and 3 , respectively). Parental map quality was further tested using R/qtl (Broman et al. 2003, script provided in Supplemental File 1). Pairwise recombination fractions demonstrated tight linkage within, but not across, different linkage groups (Supplemental Figure 4).

QTL mapping of the sex-determining locus. To verify the correctness of the linkage maps, pistillate/staminate flower data were used to map the sex-determining locus through interval mapping. Of 203 flower-bearing $F_{1}$ individuals, 101 had pistillate, 102 had staminate, and none had hermaphroditic flowers, indicating that the female parent was homozygous for the recessive female allele and the male parent was heterozygous for the dominant male allele. A single major QTL was detected at a genetic position of $21.99 \mathrm{cM}$ on chromosome 2 (chr2) in the integrated map with a peak LOD score of 60.32 (Supplemental Figure 5). This QTL (QTL.Sex) explained $80.7 \%$ of the phenotypic variance, and its localization to chr2 is in agreement with earlier reports (Dalbó et al. 2000, Riaz et al. 2006, Marguerit et al. 2009).

Characterization of the DM pathogen. The nucleotide sequence of the ITS-1 fragment amplified from the DM strain was identical to the corresponding fragment of Clade-A of the
P. viticola species complex (Supplemental Figure 6), which established it as a member of the riparia cryptic species of $P$. viticola. (Rouxel et al. 2014). To characterize the virulence of the MO-1 strain, it was used to inoculate three different grapevines: $V$. riparia Gloire de Montepellier, $M$. rotundifolia Thomas, and $V$. vinifera F2-35. M. rotundifolia Thomas appeared immune to the strain, $V$. riparia Gloire de Montpellier proved partially resistant, while $V$. vinifera F2-35 was highly susceptible, indicating that the strain represents an aggressive pathogen of cultivated grapes (Figure 1). Both parents of the $\mathrm{F}_{1}$ progeny had greater resistance to MO-1 than $V$. vinifera (Figure 1). While we consider the DM population used in this study to be a single strain, based on its appearance, we did not propagate it from a single sporangium to ensure that inoculations were done with a pure culture.

QTL mapping of DM resistance. Segregation of the DM resistance phenotype in the $F_{1}$ progeny suggested that this trait was quantitative and determined by multiple loci (Supplemental Figure 7). Of the 20 individuals in both the naturally and in vitro-infected cohorts, 16 had similarly moderate resistance ratings under both conditions. The four that differed substantially in DM coverage were all rated as highly susceptible (1 on a 1-to-10 point scale) in response to natural infection, but moderately resistant under in vitro conditions. Analysis of resistance levels in naturally-infected vines led to the detection of a major QTL at a genetic position of $12.46 \mathrm{cM}$ on chr10 in the integrated map (Rpv28.1, Figure 2). This QTL, which independently mapped to the female parent but not to the male parent, had an LOD value of 32.32 , and explained $66.5 \%$ of the phenotypic variance

Table 1 Distribution of single nucleotide polymorphism markers and the total genetic length in Kosambi distance across the Vitis rupestris B38 and Vitis riparia HP-1 parental and integrated maps.

\begin{tabular}{|c|c|c|c|c|c|c|c|c|c|}
\hline \multirow[b]{2}{*}{$\begin{array}{l}\text { Linkage } \\
\text { group }\end{array}$} & \multicolumn{3}{|c|}{ Number of markers } & \multicolumn{3}{|c|}{ Size (cM) } & \multicolumn{3}{|c|}{ Avg distance between markers (cM) } \\
\hline & $\begin{array}{l}\text { V. rupestris } \\
\text { map }\end{array}$ & $\begin{array}{l}\text { V. riparia } \\
\text { map }\end{array}$ & $\underset{\text { map }}{\text { Integrated }}$ & $\begin{array}{l}\text { map } \\
\text { mapestris }\end{array}$ & $\begin{array}{l}\text { V. riparia } \\
\text { map }\end{array}$ & $\underset{\text { map }}{\text { Integrated }}$ & $\begin{array}{l}\text { V. rupestris } \\
\text { map }\end{array}$ & $\begin{array}{l}V . \text { riparia } \\
\text { map }\end{array}$ & $\underset{\text { map }}{\text { Integrated }}$ \\
\hline LG1 & 42 & 64 & 125 & 80.5 & 94.8 & 96.0 & 0.52 & 0.68 & 0.77 \\
\hline LG2 & 50 & 43 & 99 & 64.5 & 77 & 75.2 & 0.78 & 0.56 & 0.76 \\
\hline LG3 & 51 & 57 & 115 & 65.4 & 75.7 & 77.3 & 0.78 & 0.75 & 0.67 \\
\hline LG4 & 61 & 64 & 139 & 71 & 88.9 & 87 & 0.86 & 0.72 & 0.63 \\
\hline LG5 & 63 & 59 & 139 & 71.8 & 83.5 & 82.4 & 0.88 & 0.71 & 0.59 \\
\hline LG6 & 31 & 53 & 94 & 58.6 & 86.8 & 85 & 0.53 & 0.61 & 0.90 \\
\hline LG7 & 98 & 92 & 209 & 95.9 & 116.7 & 114.6 & 1.02 & 0.79 & 0.55 \\
\hline LG8 & 57 & 50 & 119 & 75.7 & 89.8 & 88 & 0.75 & 0.56 & 0.74 \\
\hline LG9 & 66 & 37 & 110 & 63.2 & 63 & 67.8 & 1.04 & 0.59 & 0.62 \\
\hline LG10 & 65 & 33 & 107 & 65.8 & 80.9 & 74.8 & 0.99 & 0.41 & 0.70 \\
\hline LG11 & 42 & 39 & 90 & 65.2 & 75.2 & 74.4 & 0.64 & 0.52 & 0.83 \\
\hline LG12 & 68 & 71 & 163 & 87.6 & 74.3 & 90.9 & 0.78 & 0.96 & 0.56 \\
\hline LG13 & 48 & 80 & 159 & 80 & 98.3 & 96.5 & 0.60 & 0.81 & 0.61 \\
\hline LG14 & 110 & 86 & 228 & 88.4 & 96.4 & 93.9 & 1.24 & 0.89 & 0.41 \\
\hline LG15 & 55 & 54 & 117 & 65.6 & 95.7 & 93.3 & 0.84 & 0.56 & 0.80 \\
\hline LG16 & 78 & 49 & 143 & 66.3 & 82.1 & 74.5 & 1.18 & 0.60 & 0.52 \\
\hline LG17 & 41 & 53 & 102 & 64.4 & 72.7 & 71.6 & 0.64 & 0.73 & 0.70 \\
\hline LG18 & 78 & 74 & 172 & 108.7 & 125.1 & 115.3 & 0.72 & 0.59 & 0.67 \\
\hline LG19 & 73 & 57 & 153 & 62.7 & 80.5 & 75.6 & 1.16 & 0.71 & 0.49 \\
\hline Total & 1177 & 1115 & 2583 & 1401.3 & 1657.4 & 1634.1 & 0.84 & 0.67 & 0.63 \\
\hline
\end{tabular}


for disease resistance. QTL analysis of resistance levels in in vitro-inoculated leaf disks led to similar results: a significant QTL for resistance was detected at a genetic position of 15.09 $\mathrm{cM}$ on chr10 on the integrated map, explaining $24.3 \%$ of the phenotypic variance (Rpv28.2, Figure 2; mean and standard deviation scores for each genotype are reported in Supplemental Table 4). The in vitro-mapped QTL encompassed the entire Rpv28.1 interval. GBS markers that fall within the 2-LOD interval of Rpv28.1 and Rpv28.2, their LOD scores, and their projected position in the $12 \mathrm{X} . \mathrm{v} 3$ assembly of $\mathrm{V}$. vinifera (Canaguier et al. 2017) are listed (Supplemental Table 5). The 2-LOD interval surrounding Rpv28.2 is delimited by the GBS markers S10_419927 and S10_3959571, which correspond to the physical interval of chr10:419,927..3,959,571. Predicted genes within Rpv28.1 as projected to the $V$. vinifera 12X.v3 reference genome sequence are listed (Supplemental Table 6). No significant QTL for resistance was detected in the $V$. riparia parent under natural or in vitro conditions. Results of QTL analyses are summarized (Table 2) and effect plots are shown (Figure 3).

\section{Discussion}

The American grape species $V$. rupestris and $V$. riparia have adapted to disparate environmental conditions and occupy different but overlapping geographic ranges (Callen et al. 2016). They also evolved to have contrasting characteristics in dormancy, morphology, and growth habits (Munson 1909). Exploration of the genetic basis of their environmental adaptation is warranted because the viticulture industry is in need of genetic resources to mitigate the environmental impact of global climate change. The economic value of these species is evidenced by their status as cornerstone resources for developing disease-resistant, phylloxera- and stress-tolerant, fruit-bearing, and rootstock cultivars during the past century and a half (Reisch et al. 2012). Despite their proven value, $V$. rupestris and $V$. riparia have yet to be explored for their vast genetic diversity across North America. V. rupestris has been under pressure due to habitat loss and is threatened by genetic erosion (Pap et al. 2015). These conditions add urgency to a broader examination of its native populations. In a recent study, GBS markers were used to examine the genetic diversi-

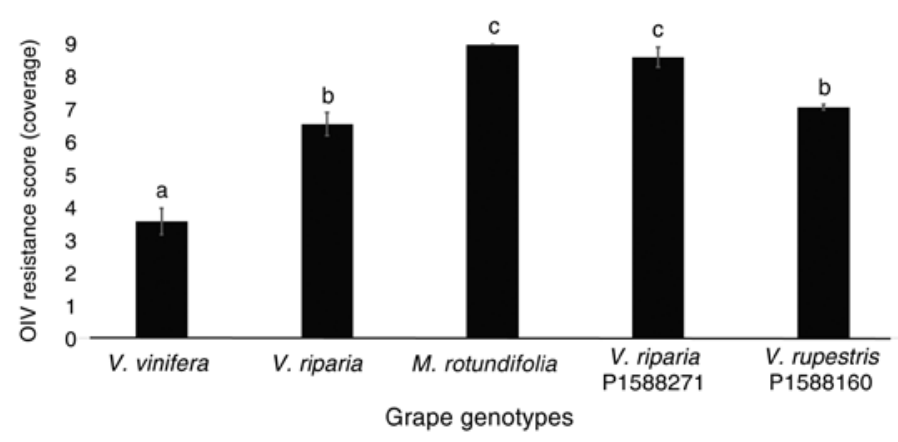

Figure 1 Resistance to Plasmopara viticola strain MO-1 in five grapevine genotypes. Level of resistance was measured as leaf area covered by sporangiophores following OIV standards in Vitis vinifera F2-35, Vitis riparia Gloire de Montepellier, Muscadinia rotundifolia Thomas, and parent genotypes V. riparia HP-1 (PI588271), and Vitis rupestris B38 (PI588160). ty of $27 \mathrm{~V}$. rupestris and $80 \mathrm{~V}$. riparia accessions housed at the USDA-ARS Grape Germplasm Collection (Klein et al. 2018). While their data are limited to accessions maintained in the repository, their work set the technological and phylogenetic foundations for a broader exploration of the natural populations of these and other wild grape relatives (Klein et al. 2018).

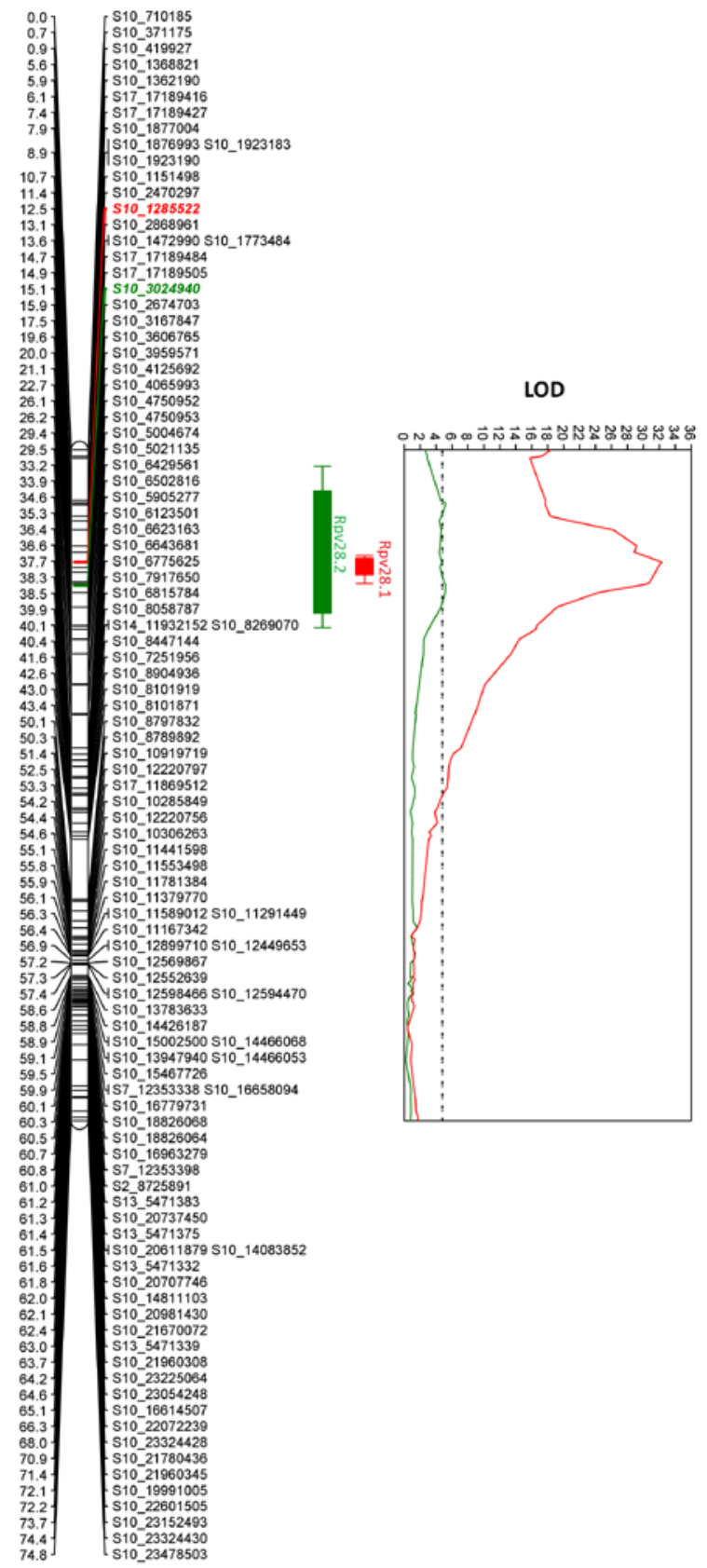

Figure 2 Quantitative trait locus analysis of downy mildew resistance across linkage group 10. The left panel represents the linkage map for LG 10 with marker ID and corresponding genetic position (cM). The right panel shows the logarithm of odds (LOD) scores obtained from interval mapping for downy mildew resistance (red: greenhouse inoculation and green: in vitro inoculation) for each marker in the integrated genetic map. The solid boundary of red and green box plots, and extreme boundary represented by their whiskers indicate 1- and 2-LOD intervals for Rpv28.1 and Rpv28.2, respectively. The horizontal black dashed-dotted line represents genome-wide LOD threshold (1000 permutations) at a $5 \%$ level of significance. Markers in red and green color on the map represent markers with the largest LOD value. 
Table 2 Summary of quantitative trait locus analysis results of the F1 hybrid progeny of Vitis rupestris B38 and Vitis riparia HP-1. LOD, logarithm of odds; DM, downy mildew.

\begin{tabular}{|c|c|c|c|c|c|c|c|c|}
\hline Trait & $\begin{array}{l}\text { Parent of } \\
\text { origin }\end{array}$ & $\begin{array}{l}\text { Peak position } \\
\text { (CM) }\end{array}$ & $\begin{array}{c}\text { Interval } \\
(\mathrm{cM})^{\mathrm{a}}\end{array}$ & $\begin{array}{l}\text { Nearest } \\
\text { marker }\end{array}$ & LOD & $\begin{array}{c}\text { LOD } \\
\text { threshold }\end{array}$ & $\begin{array}{c}\text { Explained } \\
\text { variance }(\%)^{\mathrm{c}}\end{array}$ & $\begin{array}{l}\text { Flanking } \\
\text { markers }\end{array}$ \\
\hline Sex & V. riparia & 21.99 & $21.85-22.2$ & S2_4599939 & 60.32 & 4.8 & 80.7 & $\begin{array}{l}\text { S2_3835305, } \\
\text { S2_4650244 }\end{array}$ \\
\hline $\begin{array}{l}\text { DM resistance } \\
\text { (in vitro) }\end{array}$ & V. rupestris & 15.098 & $1.8-19.8$ & S10_3024940 & 5.20 & 4.8 & 24.3 & $\begin{array}{l}\text { S17_17189505, } \\
\text { S10_2674703 }\end{array}$ \\
\hline $\begin{array}{l}\text { DM resistance } \\
\text { (in greenhouse) }\end{array}$ & V. rupestris & 12.456 & $11.86-14.85$ & S10_1285522 & 32.32 & 4.8 & 66.5 & $\begin{array}{l}\text { S10_2470297, } \\
\text { S10_2868961 }\end{array}$ \\
\hline
\end{tabular}

a2-LOD interval on the integrated genetic map.

${ }^{b}$ Genome-wide LOD threshold obtained with 1000 permutations at $p=0.05$.

'Percentage of phenotypic variance explained by quantitative trait locus.

dMarkers on each side of the largest LOD peak.
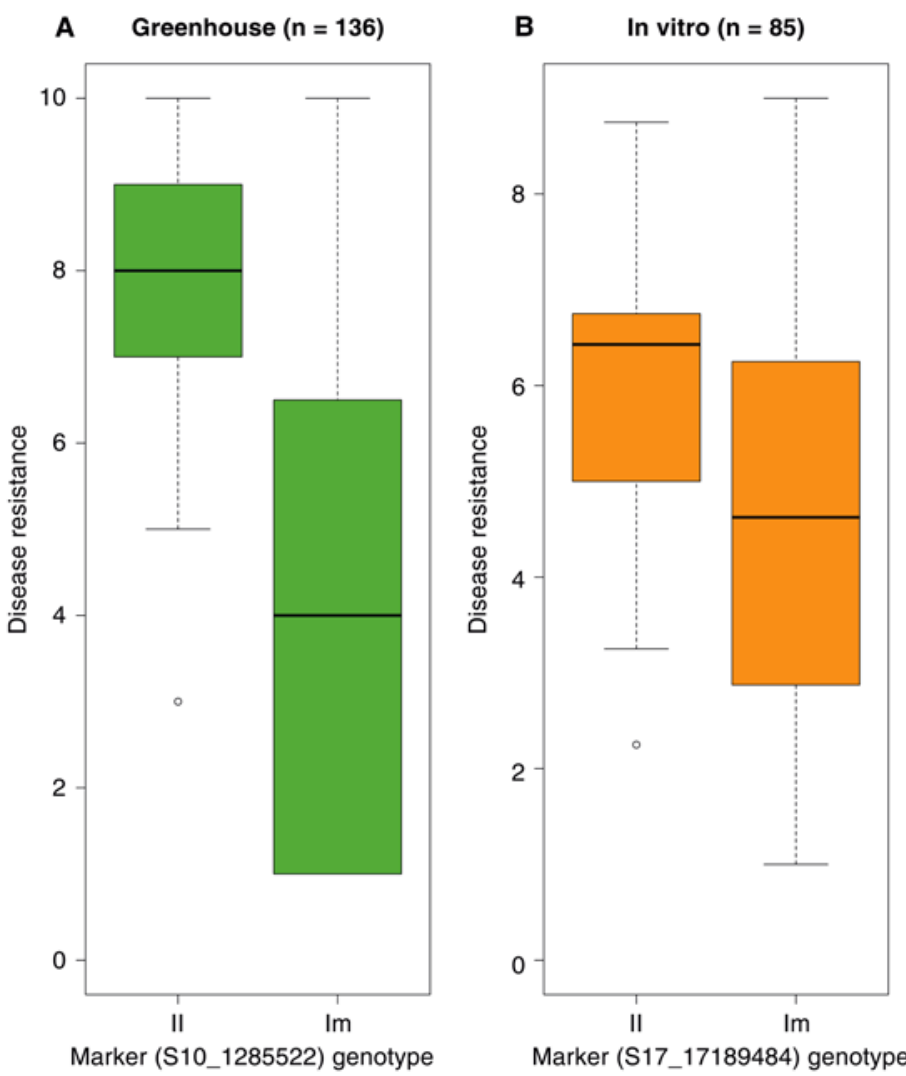

Figure 3 Effect plots showing the relative contribution to downy mildew resistance of quantitative trait locus Rpv28.1 (S10_1285522) and Rpv28.2 (S17_17189484) in the homozygous and heterozygous states.

We report here the construction of genetic linkage maps for $V$. rupestris and $V$. riparia based on an $\mathrm{F}_{1}$ population produced from a cross between these two species. The genomes of both parents had a high degree of synteny with the genome of $V$. vinifera for marker position (Supplemental Figure 3). Only $9.94 \%$ and $8.87 \%$ of $V$. rupestris and $V$. riparia markers, respectively, were assigned to a linkage group that was different from the $V$. vinifera RefSeq linkage group assignment (Supplemental Figure 2). Similarly conflicting results were reported between genetic and RefSeq positions for $18.3 \%$ and $13.7 \%$ of SNP markers in apple (Antanaviciute et al. 2012, Gardner et al. 2014). Such disagreements between linkage maps and RefSeqs do not necessarily indicate mapping errors, but may result from the presence of paralogous genomic regions or incorrect RefSeq sequence assembly. By performing a map validation step using flower sex phenotype, we verified the linkage maps we generated, since the genomic position of the sex locus is known. This gave us confidence in the accuracy of our map and the ability to reproduce the mapping of a well-known locus with our set of markers, placed as they are on the genetic maps.

Defense-related genes tend to be in the heterozygous state in plants (McDowell and Simon 2006), and genes that confer resistance to the same pathogen are often located at different loci in various grape genotypes (Gadoury et al. 2012, Buonassisi et al. 2017). Consequently, two resistant grapevine parents will likely produce an $\mathrm{F}_{1}$ population in which defense-related traits will segregate, as recently demonstrated (Divilov et al. 2018). We followed a similar approach as described by Divilov et al. (2018), in that we established an $\mathrm{F}_{1}$ hybrid population from a cross between two DM-resistant accessions. DM resistance segregated in the $\mathrm{F}_{1}$ progeny, which enabled us to map a major QTL (LOD of 32.32), Rpv28.1, in the female parent that accounted for $66.5 \%$ of the phenotypic variance in naturally infected plants under greenhouse conditions. Repeating this analysis using a leaf disk DM inoculation assay led to mapping of another resistance QTL from the female parent, Rpv28.2, which overlaps with Rpv28.1, confirming the contribution of this locus to defense against DM. This QTL, however, explained only $24.3 \%$ of the phenotypic variance and had an LOD value of 5.2, indicating that the trait is strongly influenced by the environment. Other possible reasons for the lower LOD value with respect to the QTL detected in the greenhouse assay might include limited population size and/or phenotyping errors. Notably, of the 20 vines shared between the naturally infected and the in vitro-inoculated cohorts, 16 had similar and four had different phenotypes. All four of the latter were rated highly susceptible in the greenhouse, but moderately resistant in vitro. Unfortunately, the number of plants was too low to determine why these four individuals had so much lower resistance in the greenhouse. No QTL were identified from the male parent, which is surprising given the results of our virulence assay on multiple species (Figure 1), in which the $V$. riparia parent showed even greater resistance to this DM strain than the $V$. rupestris parent. One possible reason for this finding 
may be the presence of multiple components of resistance that contribute at low levels to the observed resistance phenotype in the male parent, which were below the threshold of detection in the offspring. Other possibilities include a major QTL that was present in a homozygous state or in a region of the genome with low marker coverage. We report here the identification of two overlapping DM resistance loci, Rpv28.1 and Rpv28.2. While these may in fact represent a single resistance locus, we identified them in separate assays that produced different LOD scores, different levels of variance explained, and different numbers of markers included under the peaks. Therefore, we think it more prudent to retain separate nomenclature for these loci.

While the high LOD value for Rpv28.1 and its reproducibility lend strong support for the presence of a resistance QTL on chr10, our experiments have limitations. Importantly, resistance was likely assessed against a single strain of $P$. viticola. Furthermore, our results lack multi-season field data. At the time of writing, the entire $\mathrm{F}_{1}$ population has been established in the field in both New York and Missouri. Both locations have high DM disease pressure, but represent different climates where the prevailing DM populations are likely dominated by different cryptic species of $P$. viticola (Rouxel et al. 2014). In the future, it will be important to test the resistance of this progeny under vineyard conditions. The New York and Missouri plantings will enable us to collect data on how various $P$. viticola strains and climatic conditions influence the performance of this QTL in the field.

Rpv28.1 is responsible for $66.5 \%$ of resistance against an aggressive DM pathogen and, therefore, it may be used for breeding grape cultivars with reduced requirement for fungicide input. The applicability of this locus is even more relevant because, to our knowledge, it is the first defense-related QTL in this region of chr10. Previously, it was hypothesized on the basis of gene expression measurements that DM resistance in the hybrid cultivar Regent was encoded by three CC-NBSLRR-type resistance genes on chr10 (Kortekamp et al. 2008). However, these genes mapped to a physical distance of at least $13 \mathrm{Mb}$ away from Rpv28.1 and Rpv28.2, near the end of the opposite arm of chr10. Interestingly, the DM-resistant parent, $V$. rupestris $\mathrm{B} 38$, harbored another QTL for DM resistance, Rpv19 on chromosome 14, different from the QTL identified in our study (Divilov et al. 2018). We detected no QTL for DM resistance at this locus in either the greenhouse or in vitro assays performed in this study. The most likely reason that we did not observe this QTL is that the DM isolate in this study was different from the isolate that elicits disease resistance conveyed by Rpv19. Additionally, Rpv19 resistance is characterized by a hypersensitive reaction that was not observed in the Rpv28 resistance phenotype. Therefore, $V$. rupestris B38 is a promising source for multiple resistance loci and may prove a useful component of "gene pyramiding" schemes. The premise of gene pyramiding is that combining various defense mechanisms against the same class of pathogen will result in more stable resistance than introgression of a single resistance gene, particularly when considering the ability of different resistance gene products to recognize different pathogen iso- lates. Based on insight into the evolution of $\mathrm{R}$ genes in several plant species (McDowell and Simon 2006), it is not surprising that $V$. rupestris $\mathrm{B} 38$ carries several resistance factors against the same pathogen. The combination of potentially multiple resistance mechanisms represented by Rpv19 and Rpv28, afford protection against different isolates of the pathogen and provide a survival advantage in nature. Future work will assess the virulence of other DM isolates from different $P$. viticola clades on Rpv28 DM-resistant individuals to ascertain the breadth of recognition for this locus.

Introgression of several loci to provide resistance against the same pathogen is only possible with marker-assisted selection (MAS). The GBS markers that define the new QTL may prove valuable for the development of molecular markers for MAS (Table 2 and Supplemental Table 5). Only 24.3\% of SNPs segregated within both $V$. vinifera and wild Vitis germplasm (Myles et al. 2010), suggesting that a portion of the heterozygous $V$. rupestris GBS markers can be selected readily when this resistance haplotype is introgressed into a predominantly $V$. vinifera background. Although SNP-based genotyping has gained popularity in grape breeding, many breeding programs still rely on simple sequence repeat (SSR) markers. A significant number of SSR markers are transferable from $V$. vinifera to wild Vitis species and hybrid grapes (Garris et al. 2009, Pap et al. 2015, Hammers et al. 2017). We identified six SSR markers that fall within or closely flank the region spanning Rpv28.1 and Rpv28.2 (Supplemental Table 7). These markers were developed originally for $V$. vinifera, and their applicability and polymorphism in $V$. rupestris $\mathrm{B} 38$ remains to be tested. Because $V$. rupestris $\mathrm{B} 38$ is now known to harbor multiple DM resistance genes (Rpv19 and Rpv28), marker-assisted selection is essential to identify which resistance alleles are passed to its progeny.

Highly effective MAS, however, will require development of markers closely linked to Rpv28.1. The strong synteny (Supplemental Figure 3) and partial conservation of SSR markers between $V$. vinifera and $V$. rupestris indicate that designing SSR primers based on the orthologous $V$. vinifera sequences may be a workable, though potentially ineffective, way to achieve this goal. A genomic library or a genome assembly of $V$. rupestris would make this approach more fruitful. Other marker types, including rhAmpSeq and KASP, could also be useful for this purpose and for further mapping work with this population. In addition, it may prove useful for future breeding efforts to survey the extant grapevine germplasm repositories for the markers associated with Rpv28 resistance and assess allelic diversity at this locus. Considering the importance of this species as a resource for grape breeding, the establishment of genomic tools would be a well-justified investment for the grape research community.

\section{Conclusion}

The hypothesis that a $V$. rupestris $\times V$. riparia $\mathrm{F}_{1}$ progeny can facilitate mapping of economically relevant loci was supported by the identification of the DM resistance QTL Rpv28.1 and Rpv28.2 in the V. rupestris genome. The novelty of this resistance locus suggests that the biological diversity 
of North American Vitis remains an extensive and still largely unexplored resource for grapevine breeding. This paper and its supplemental material provide a valuable resource for grape breeders, geneticists, and those teaching genetic mapping in an outcrossing species.

\section{Literature Cited}

Agurto M, Schlechter RO, Armijo G, Solano E, Serrano C, Contreras RA, Zúñiga GE and Arce-Johnson P. 2017. RUN1 and REN1 pyramiding in grapevine (Vitis vinifera $\mathrm{cv}$. Crimson Seedless) displays an improved defense response leading to enhanced resistance to powdery mildew (Erysiphe necator). Front Plant Sci 8:758.

Alleweldt G and Possingham JV. 1988. Progress in grapevine breeding. Theor Appl Genet 75:669-673.

Antanaviciute L et al. 2012. Development of a dense SNP-based linkage map of an apple rootstock progeny using the Malus Infinium whole genome genotyping array. BMC Genomics 13:203.

Broman KW, Wu H, Sen Ś and Churchill GA. 2003. R/qt1: QTL mapping in experimental crosses. Bioinformatics 19:889-890.

Buonassisi D et al. 2017. Breeding for grapevine downy mildew resistance: A review of "omics" approaches. Euphytica 213:103.

Callen ST, Klein LL and Miller AJ. 2016. Climatic niche characterization of 13 North American Vitis species. Am J Enol Vitic 67:339-349.

Canaguier A et al. 2017. A new version of the grapevine reference genome assembly (12X.v2) and of its annotation (VCost.v3). Genom Data 14:56-62.

Dalbó MA, Ye GN, Weeden NF, Steinkellner H, Sefc KM and Reisch BI. 2000. A gene controlling sex in grapevines placed on a molecular marker-based genetic map. Genome 43:333-340.

Danecek P et al. 2011. The variant call format and VCFtools. Bioinformatics 27:2156-2158.

Di Gaspero G et al. 2012. Selective sweep at the Rpv3 locus during grapevine breeding for downy mildew resistance. Theor Appl Genet 124:277-286

Divilov K, Barba P, Cadle-Davidson L and Reisch BI. 2018. Single and multiple phenotype QTL analyses of downy mildew resistance in interspecific grapevines. Theor Appl Genet 131:1133-1143.

Duitama J, Quintero JC, Cruz DF, Quintero C, Hubmann G, FoulquiéMoreno MR, Verstrepen KJ, Thevelein JM and Tohme J. 2014. An integrated framework for discovery and genotyping of genomic variants from high-throughput sequencing experiments. Nucleic Acids Res 42:6.

Elshire RJ, Glaubitz JC, Sun Q, Poland JA, Kawamoto K, Buckler ES and Mitchell SE. 2011. A robust, simple genotyping-by-sequencing (GBS) approach for high diversity species. PLoS ONE 6:e19379.

Feechan A et al. 2013. Genetic dissection of a TIR-NB-LRR locus from the wild North American grapevine species Muscadinia rotundifolia identifies paralogous genes conferring resistance to major fungal and oomycete pathogens in cultivated grapevine. Plant J 76:661-674.

Gadoury DM, Cadle-Davidson L, Wilcox WF, Dry IB, Seem RC and Milgroom MG. 2012. Grapevine powdery mildew (Erysiphe necator): A fascinating system for the study of the biology, ecology and epidemiology of an obligate biotroph. Mol Plant Pathol 13:1-16.

Gardner KM, Brown P, Cooke TF, Cann S, Costa F, Bustamante C, Velasco R, Troggio M and Myles S. 2014. Fast and cost-effective genetic mapping in apple using next-generation sequencing. G3 (Bethesda) 4:1681-1687.

Garris A, Clark L, Owens C, McKay S, Luby J, Mathiason K and Fennell A. 2009. Mapping of photoperiod-induced growth cessation in the wild grape Vitis riparia. J Am Soc Hortic Sci 134:261-272.
Germplasm Resources Information Network. Beltsville, MD: United States Department of Agriculture, Agricultural Research Service. Accessed 28 Nov 2019. https://www.ars-grin.gov/.

Glaubitz JC, Casstevens TM, Lu F, Harriman J, Elshire RJ, Sun Q and Buckler ES. 2014. TASSEL-GBS: A high capacity genotyping by sequencing analysis pipeline. PLoS ONE 9:e90346.

Grattapaglia D and Sederoff R. 1994. Genetic linkage maps of Eucalyptus grandis and Eucalyptus urophylla using a pseudo-testcross: Mapping strategy and RAPD markers. Genetics 137:1121-1137.

Grimplet J, Van Hemert J, Carbonell-Bejerano P, Díaz-Riquelme J, Dickerson J, Fennell A, Pezzotti M and Martínez-Zapater JM. 2012. Comparative analysis of grapevine whole-genome gene predictions, functional annotation, categorization and integration of the predicted gene sequences. BMC Res Notes 5:213-222.

Hammers M, Sapkota S, Chen LL and Hwang C-F. 2017. Constructing a genetic linkage map of Vitis aestivalis-derived "Norton" and its use in comparing Norton and Cynthiana. Mol Breeding 37:64.

Hyma KE, Barba P, Wang M, Londo JP, Acharya CB, Mitchell SE, Sun Q, Reisch B and Cadle-Davidson L. 2015. Heterozygous mapping strategy (HetMappS) for high resolution genotyping-by-sequencing markers: A case study in grapevine. PLoS ONE 10:e0134880.

International Organization of Vine and Wine. 2009. Technical Standards and Documents, 2nd ed. Organisation Internationale de la Vigne et du Vin, Paris, France.

Jaillon $\mathrm{O}$ et al. 2007. The grapevine genome sequence suggests ancestral hexaploidization in major angiosperm phyla. Nature 449:463-467.

Klein LL, Miller AJ, Ciotir C, Hyma K, Uribe-Convers S and Londo J. 2018. High-throughput sequencing data clarify evolutionary relationships among North American Vitis species and improve identification in USDA Vitis germplasm collections. Am J Bot 105:215-226.

Kortekamp A, Welter L, Vogt S, Knoll A, Schwander F, Töpfer R and Zyprian E. 2008. Identification, isolation and characterization of a CC-NBS-LRR candidate disease resistance gene family in grapevine. Mol Breeding 22:421-432.

Li H and Durbin R. 2009. Fast and accurate short read alignment with Burrows-Wheeler transform. Bioinformatics 25:1754-1760.

Liang $\mathrm{Z}$ et al. 2019. Whole-genome resequencing of 472 Vitis accessions for grapevine diversity and demographic history analyses. Nat Commun 10:1190.

Marguerit E et al. 2009. Genetic dissection of sex determinism, inflorescence morphology and downy mildew resistance in grapevine. Theor Appl Genet 118:1261-1278.

McDowell JM and Simon SA. 2006. Recent insights into R gene evolution. Mol Plant Pathol 7:437-448.

Migicovsky Z et al. 2016. Genomic ancestry estimation quantifies use of wild species in grape breeding. BMC Genomics 17:478.

Munson TV. 1909. Foundations of American Grape Culture. TV Munson and Son, Texas.

Myles S, Chia J-M, Hurwitz B, Simon C, Zhong GY, Buckler E and Ware D. 2010. Rapid genomic characterization of the genus Vitis. PLoS ONE 5:e8219.

Ouellette LA, Reid RW, Blanchard SG and Brouwer CR. 2018. LinkageMapView-rendering high-resolution linkage and QTL maps. Bioinformatics 34:306-307.

Pap D, Miller AJ, Londo JP and Kovács LG. 2015. Population structure of Vitis rupestris, an important resource for viticulture. Am J Enol Vitic 66:403-410.

Reisch BI, Owens CL and Cousins PS. 2012. Grape. In Fruit Breeding. Handbook of Plant Breeding, vol 8. Badenes ML and Byrne DH (eds.), pp. 225-262. Springer, Boston, MA. 
Riaz S, Krivanek AF, Xu K and Walker MA. 2006. Refined mapping of the Pierce's disease resistance locus, PdR1, and Sex on an extended genetic map of Vitis rupestris $\times V$. arizonica. Theor Appl Genet 113:1317-29.

Riaz S, Tenscher AC, Graziani R, Krivanek AF, Ramming DW and Walker MA. 2009. Using marker-assisted selection to breed Pierce's disease-resistant grapes. Am J Enol Vitic 60:199-207.

Riaz S, Pap D, Uretsky J, Laucou V, Boursiquot JM, Kocsis L and Walker AM. 2019. Genetic diversity and parentage analysis of grape rootstocks. Theor Appl Genet 132:1847-1860.

Rouxel M et al. 2014. Geographic distribution of cryptic species of Plasmopara viticola causing downy mildew on wild and cultivated grape in eastern North America. Phytopathology 104:692-701.
Van Ooijen JW. 2006. JoinMap 4: Software for the calculation of genetic linkage maps in experimental populations of diploid species. Kyazma BV, Wageningen, The Netherlands.

Van Ooijen JW. 2009. MapQTL 6: Software for the mapping of quantitative trait loci in experimental populations of diploid species. Kyazma BV, Wageningen, The Netherlands.

Voorrips RE. 2002. MapChart: Software for the graphical presentation of linkage maps and QTLs. J Hered 93:77-78. 